\title{
ACERCA DE LOS APORTES DE ACIDO ASCORBICO EN EL FETO Y RECIEN NACIDO
}

\author{
Por la Dra. BLANCA MONTERO SIERRA \\ (Resumen de la Memoria de Prueba).
}

\section{Introducción}

El conocimiento de la vitamina $C$ como entidad quimica, ha permitido estudiar con exactitud su influencia sobre los organismos vivos. Los diversos problemas planteados en este. terreno, han motivado muchos trabajos de orden clínico y experimental. De entre ellos me interesaron especialmente los relacionados con las necesidades de vitamina $C$ en el recién nacido $\mathrm{y}$ lactante, por cuanto los suplementos de ácido ascórbico, se consideraban innecesarios durante el primer semestre de la vida, dada la resistencia al escorbuto que se observa en este período. La explicación de este hecho era para algunos, la capacidad del organismo joven para sintetizar esta sustancia; para otros, la existencia de reservas que el niño tràe al nacer.

En otras palabras, se observaba que las criaturas eran refractarias a la carencia. pero se discutía el por qué de esta cualidad.

Llevando como mira el tratar de afirmar o negar la posibilidad de una sintesis orgánica de ácido cevitamínico diutante los primeros meses de la vida, he tenido que concretarme a estudiar los valores de la sangre materna, leche, placenta, sangre del cordón y sangre del recién nacido y lactante. Las relaciones que estas cifras presentan, aunque no permitan dilucidar en definitiva el problema enunciado, proporcionan algunos datos útiles que pueden orientar hacia una dietética más racional, sobre todo en el lactante alimentado artificialmente. 


\section{PRIMERA PARTE}

Son de todos convidos los trabajos que permitieron la identificación y posteriormente la síntesis y dosificación del ácido ascórbico; asi como los que se han efectuado en el laboratorio para llegar a determinar la dosis preventiva y curativa del escorbuto experimental.

Brevemente se exponen a continuación los métodos que permiten aplicar estas adquisiciones a la clínica.

Desde que se identificó el escorbuto como una enfermedad por carencia de alimentos frescos, hasta el momento actual in que se prepara sintéticamente el principio activo, ha transcartido una larga etapa que ha modificado los conceptos cliricos. Durante la época en que se tenía sólo el conocimiento empírico del fenómeno, se anotaba la diferençia entre el individuo escorbútico y el que no lo era, considerando a este último como sano: cuando fué descubierto el valor de los alimentos frescos y de los jugos de frutas frente al escorbuto, el problema pareció resuelto; era muy fácil curar el cuadro establecido, y prevenirlo alimentado a los individuos amenazados con verduras y frutas frescas.

Posteriormente, gracias a la facilidad con que se dosifica el ácido ascórbico con la técnica ideada por Tíllmans. el antiguo concepto ha sido sustituído por otro más amplio que considera entre los dos extremos de: salud perfecta y estado caren. cial, el grupo enorme de intermediarios que comprende desde la hipovitaminosis hasta el escorbuto larvado. Estos estados cquivalen al período de latencia del escorbuto experimental: pueden durat largo tiempo, mejorar o agravarse según las condiciones del régimen; representan la etapa que recorre el organismo desde el estado de equilibrio hasta el de total carencia que se señala por la aparición del escorbuto.

Precisamente es ésta la época de mayor interés clínico. puesto que permite prevenir la aparición de un cuadro grave. Observando cuidadosamente a los individuos que presentan hipovitaminosis se encuentran síntomas vagos como astenia. anorexia y lesiones gingivales esbozadas; en los niños el cuadto resulta más complejo por la repercusión que trae la falta de apetito, sobre la curva de peso, y por un descenso de la inmunidad.

Para facilitar el estudio clínico de los estados de hipovitaminosis se ha ideado una serie de métodos sencillos, que, unidos al examen general del individuo, permiten orientarse en el sentido de un cuadro carencial. Ellos son por ejemplo: 
a) La inyección intradérmica de una solución de diclorofenol indofenol: se deduce el estado de saturación del orgárismo, del tiempo que tarda en decolorarse (reducirse), la sustancia mediante el ácido ascórbico de los téjiclos.

b) Prueba de la fragilidad vascular (Rumpel-Leede). haciendo la compresión de los vasos de un miembro mediante un manguito: se observa el número de petequias que aparecen en el segmento distal en una superficie dada.

c) Prueba de la resistencia capilar (Göthlin) se bace el vacio en un segmento de la piel por medio de una copa; lo mismo que en el caso anterior, se cuentan las petequias.

Estos métodos son muy útiles pata señalar a grandes rasgos el estado nutritivo de los individuos bajo el punto de vista de Vitamina $C$. pero en ningún caso pueden considerarse métodos muy sensibles ni precisos. Así, los dos últimos son positivos sólo cuando los valores sanguíneos descienden a 0, $14 \mathrm{mgr}$., valor excesivamente bajo. Aun con estos métodos, Göthlin (5) pudo demostrar un alto porcentaje de individuos que sufrían hipovitaminosis entre los niños pobres de Suecia y N. York. Otro hecho que ha venido a facilitar los estudios clínicos sobre factor $C$. es la comprobación de esta sustancia en la orina.

Mediante el estudio sistemático de la excreción urinaria en individuos sanos, se ha podido establecer, primero por Harris 137) $y$, posteriormente, en muchos otros trabajos, la diferencia entre el estado de equilibrio y el estado de saturación. En El primero caben dos grupos de individuos:

a) Los que con una excreción de $10 \mathrm{mgrs}$. diarios, límite inferior de la normal. no presentan ningún signo patológico.

b) Los que mantienen una excreción mediana de 20 mgrs. diarios que refleja una buena ingestión.

El segundo grupo está formado por los individuos que excretan diariamente 40 mgrs., son los que están saturados.

El minimum óptimo de ingestión. según estos trabajos. es $25 \mathrm{mgrs}$. diarios.

Además del simple estudio de la excreción por la orina se completaron estas experiencias, dando dosis elevadas de ácido ascórbico durante varios días: se comprobó un comportamiento muy diferente para cada uno de los grupos anteriores. Los individuos saturados, responden al sobrecargo con un aumento inmędiato de la excreción por la orina, aumento que equivale al $50 \%$ de la dosis de lujo. Los del grupo b) tardan 
algunos días en aumentar su excreción. pero terminan Dor colocarse en las mismas condiciones que los anteriores.

Finalmente, los del grupo a) demoran un tiempo mucho nas largo en excretar la sustancia recibida.

La determinación paralela en sangre y orina hecha después por Neuweiler (37), Baumann y Rappolt (4), demostró también que era tanto más difícil saturar a un individuo. cuanto más bajos eran los valores de ácido cevitamínico en la sangre y orina,

Se interpretan estos hechos, aceptando para los casos de excreción mínima y mediana. un equilibrio que puede mantererse siempre que no interfieran factores extraños. Para el grupo de los saturados, la situación es óptima bajo el punto de vista factor $C$., ya que les permite en un momento dado hacer frente a gastos extraordinarios sin que su organismo se resienta.

Todos estos hechos tienden a afianzar la idea de que el hombre necesita tecibir cantidades suficientes de factor $C$, porque la carencia de él se manifiesta por estados de hipovitaminosis cuando no de escorbuto franco.

La síntesis de la vitamina $C$ por las suprarrenales buma. nas o por cualquier otro órgano, parece cada vez menos probable; hoy se interpreta el contenido alto de ellas como dependiente de su metabolismo intenso, puesto que esta sustancia actúa como un transportador de O. Roe y Baumann (38) han comprobado la existencia de un fermento, capaz de reducir la forma oxigenada del ácido ascórbico que se encuentra en la sangre humana y que seguramente juega un papel importante en las óxidos reducciones biológicas.

Es uniformemente aceptado que la edad en que se presenta el escorbuto en el niño, es sobre los seis meses y hasta los dos años. La opinión que estos hechos merecen a los pediatras se expresa en el párrafo de Meyer y Nassau (25) que se transcribe textualmente: 'El que el escorbuto jamás se presente antes del $5 .^{\circ}$ mes y que requiera un plazo preparatorio de 2-3 meses como máximo, permite prescindir de zumos vitamínicos durante los primeros meses, pero no debe retrasarse su administración más allá del cuarto mes. Debe recomendarse su empleo tanto en los niños alimentados con biberón como en los criados al pecho. Después de la lactancia, al atenuarse la intensidad de desarrollo y crecimiento, parecen disminuir también gradualmente los requerimientos vitamínicos, tesultando suficientes cantidades menores de jugos de fruta". 
Al mismo tiempo, es corriente la observación de niños que, a pesar de una alimentación defectuosa bajo el punto de vista factor $C$ se desatrollan en buenas condiciones.

Estas observaciones llevaron a afirmar que el recién nacido presenta ésta defensa al escorbuto, porque es capaz de sintetizar factor C. Giroud (9) y sus colaboradores opinàn que: el recién nacido representa sólo una fase de evolución orgánica que dura toda la vida; el feto poseeria la capacidad máxima de sintetizar ácido ascórbico, esta facultad se atenúa en el recién nacido y se pierde a través de la infancia para terminar siendo eì adulto un organismo carenciable.

El análisis de estas consideraciones sugería como tema de estudio, la investigación de vitamina $C$ en los recién nacidos y con las madres, tratando de aclarar las relaciones de interés que en ellos se encontraran.

\section{PARTE EXPERIMENTAL}

Exposición del método.

Tomando en cuenta que la lactancia es en cierto sentido la continuación de la preñez, ya que el recién nacido depende estrechamente del organismo materno mientras dura la alimentación natural, se empezó por estudiar los valores habituales de la sangre en mujeres en el último periodo del em: barazo.

Se hizo la dosificacion por el método de Tillmans: usando para preparar el colorante, comprimidos Merck de diclorofenol indofenol que equivalen a $1 \mathrm{mgr}$. de ácido ascórbico, cuyo contenido se verificó usando una solución de colo. rante en polvo y otra de ácido ascórbico recién titulada. La selución se preparaba al $2 . \%$ y se usaba durante 6 días como plazo máximo.

Las titulaciones se hicieron exclusivamente en sangee total, usando para la defecación ácido tricloracético al $20 \%$ Se procedió en la forma siguiente:

$\begin{array}{lllllll}\text { sangre total } & \ldots & \ldots & \ldots & \ldots & \\ \text { ácido tricloracético al } & 20 & \% & \ldots & 2 & \mathrm{cc} \\ \text { agua destilada } & \ldots & \ldots & \ldots & \ldots & \ldots & 6 \\ \mathrm{cc} .\end{array}$

Se agita y se filtra: $5 \mathrm{cc}$. del filtrado corresponden a $1 \mathrm{cc}$. de sangre total.

Las muestras se recogian con floruro de sodio y se tuvo especial precanción de titular inmediatamente de tal manera 
que el factor oxidación por el medio ambiente no debe haber interverido como elemento de error.

Se prefirió la dosificación en sangre por consideraria más exacta que la que puede deducirse de la excreción urinaria, susceptibles de modificarse incluso por los estados de funcionalismo renal y además, porque los casos en que se iba a realizar el trabajo se prestaban muy poco a las determinacio. nes seriadas y largas que se necesitan en el estudio del contenido urinario; los servicios de maternidad son de urgencia, las mujeres permanecen pocos días y llegan pocas horas antes del parto, cuando el embarazo se ha desarrollado en condiciones normales.

La titulación en leche y tejido placentario se hizo en forma análoga a la sangre:

$\begin{array}{llllllll}\text { placenta } & \ldots & \ldots & \ldots & & & & \\ \text { ácido tricloracético al } & 20 & \% & \ldots & 2 & \mathrm{gc} . \\ \text { agua destilada } & \ldots & \ldots & \ldots & \ldots & \ldots & 6 & \mathrm{cc} .\end{array}$

Se macera en un mortero de vidrio durante algunos minutos hasta reducir a una lámina el trozo de tejido; se filtta y se usan $5 \mathrm{cc}$. del filtrado refiriéndolos a $1 \mathrm{gr}$. de placenta. Para la leche se usó el mismo procedimiento y no se encontró dificultad en el filtrado de ella, salvo algunos casos raros en que aparecía opalescencia muy marcada: en general ésta desaparecía filtrando de nuevo.

\section{2) ¿Existe una hipovitaminosis del embarazo?}

Los valores encontrados en embarazadas de término fueron sensiblemente iguales a los obtenidos en adultos de ambos sexos en diferentes condiciones fisiológicas; en 10 casos se obtuvo un término medio de $1,09 \mathrm{mgr}$. \% ce. $\pm 0,3$.

No se comprobó, pues, en estos casos el descenso de que Jabla Neuweiler (31). No puede relacionarse tampoco-este resultado, con el que obtuvieron Törõk y Neufeld (40), porque ellos usaton un método indirecto y no dan cifras; clos encuentran que el $23 \%$ de las embarazadas presentan valores subnormales.

Para pronunciarse sobre el estado de saturación de las madres era necesario hacer pruebas de sobrecargo que no se efectuaron, por la dificultad del control urinario en las puérperas y porque no era un dato de gran importancia, dada la índole de este trabajo. 
Se buscó en seguida la cifra correspondiente en el cordón, tomando la muestra del cabo central antes de que se produjera el alumbramiento; se encontró siempre algo superior:

Sangre materna: 1 mgr. $\% \pm 0.3$ (T. M. 18 casos)

Sangre del cordón: $1,7 \mathrm{mgr}$. \% \pm 0.3 (T. M. 26 casos)

\section{GRAFICO $\mathrm{N}^{\circ} 1$}

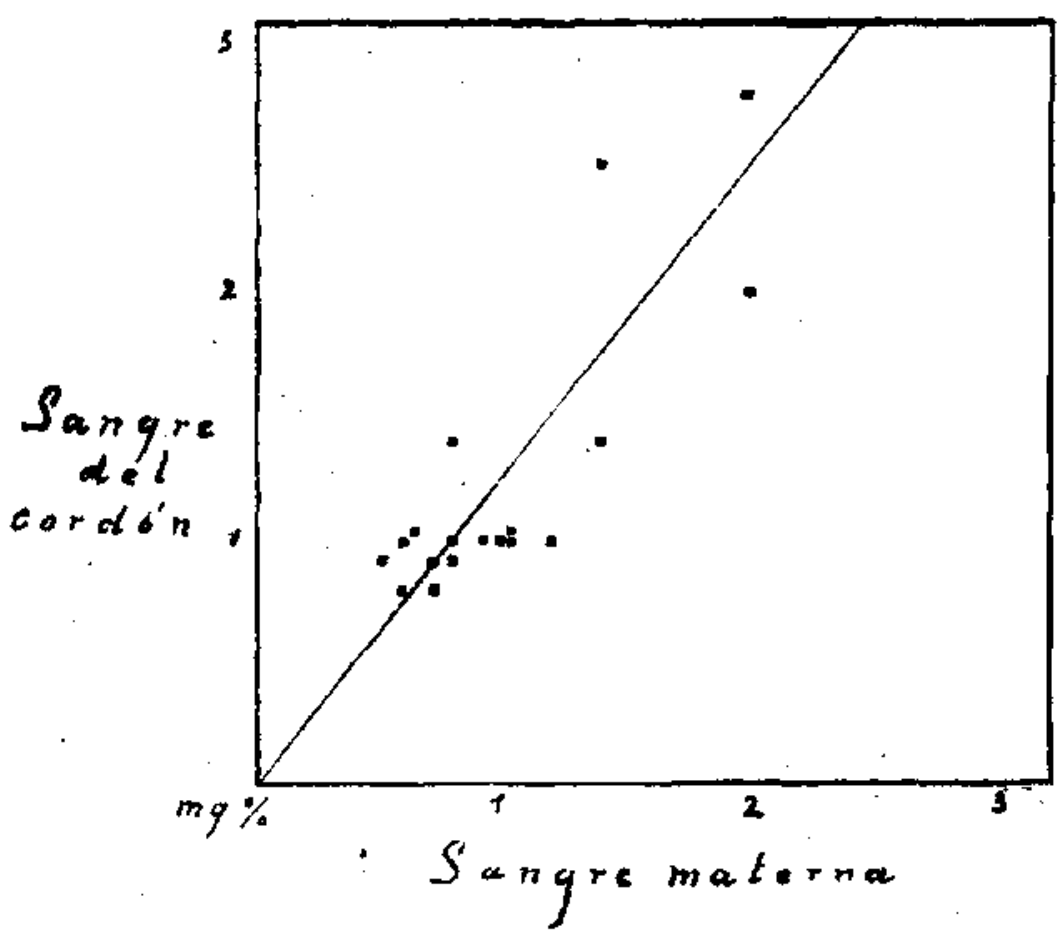

Posteriormente, con el objeto de explicar esta diferencia. se hizo la dosificación de factor $C$, en el tejido placentario y se éncontraron esta vez, cifras francamente superiores a las de la sangre:

Término medio; $1.8 \mathrm{mgr}$. \% por cc, en el cordón.

Término medio: $10,2 \mathrm{mgr}$. \% en la placenta en gr. $\pm 2,1$. (13 casos). 


\section{GRAFICO N.9 2.}

Punto, igual a Sala comín.

Triángulo, igual a Pensionado.

Cruz. igual a Sobrecargo.

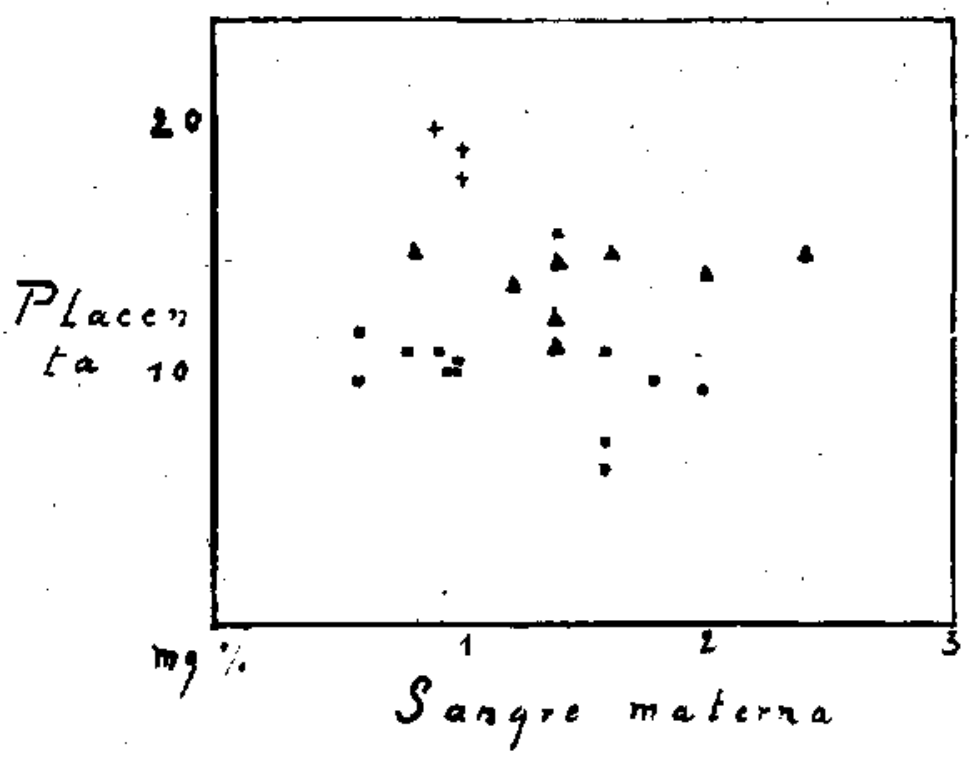

En los casos del pensionado, el porcentaje se tomó aparte: la titulación, en la mayoría de estos casos, fué hecha varias horas clespués del parto, encontrándose $1,5 \mathrm{mgr}$. en el cordón y 13,8 mgrs, en la placenta. (término medio). Los valores pueden haber descendido por el contacto con el aire, pero a pesar de esto son superiores en la placenta a los dados por las madres de sala común.

Comparando este valor con los encontrados en sangre materna y sangre del cordón: 0,96 y $1.6^{2}$ mgr. respectivamente (expresado en gr.), se encuentra que es 10 veces superior a la primera y 6 veces a la segunda.

Parece probable entonces que la concentración más alta encontrada en el cordón tenga como causa la riqueza en vitamina $C$ del tejido placentario.

Neuweiler ha encontrado en sangre del cordón un término medio de 2 mgrs., y tomando aisladamente sangre de la arteria umbilical encuentra valores más bajos $(1,2)$ que en la sangre de la vena $(2,8)$. El autor atríbuye a la congestión que produce trabajo, el valor alto obtenido en la vena umbilical. En ese mismo trabajo se expresa la idea que el feto 
toma de la placenta ácido ascórbico, porque existe un alto contenido en la placenta. No se refiere a cifras determinadas.

\section{3). ¿Existe una sintesis plarentaria?}

La comprobación de los valores altos en la placenta, ha planteado la cuestión de si éste es un órgano capaz de sintetizar factor $C$, o si simplemente en ella se acumula, sirviendo en este caso de reservorio al feto para suministrarle los aportes necesarios. Sin poder responder categóricamente a ninguna de las hipótesis sugeridas, parece más probable que se trate de пra fijación electiva como se ha llamado a este proceso: el tejido placentario tendria una especial avidez por el factor $C$. lo que permitiría un acúmulo de esta sustancia a su nivel. La reserva de 56 mgrs. que resulta el término medio para placentas de 500 grs., representa un valor considerable, sobre todo si se compara con el que se encuentra en aquellos órganos seña. lados como los más ricos en ácido ascórbico: la hipófisis 0,56 mgr. (valor total) y las suprarrenales $10 \mathrm{mgrs}$. (id.), dado su escaso peso.

La clínica y los trabajos experimentales parecen apoyat la hipótesis de una acumulación placentaria: en efector se citan casos de escorbuto durante el embarazo, Gaethgens (7), el cuadro apareció con ocasión de un trastorno gastro intestinal. de la madre (factor desencadenante).

En este trabajo, por dificultades insubsanables, sólo se hicieron tres pruebas de sobrecargo: durante la espera, se hizo una inyección de Cebión intramuscular de 300 mgrs. Produ: cido el parto, se encontró en estas madres los valores siguientes:

CUADRO N. ${ }^{\circ} 1$.

Sobrecargo de Vitamina $C$.

Observacione:

A. $\mathrm{O}$

R. P.

C. V.
Acido arcórbico mgr. \%

Cordón

1.19

0,9

$1 \quad 18$

Diferencia en el contenido del cordón y de la placenta.

$O$ sea, los valores en el cordón se mantienen, siendo más bien bajos, en cambio, la placenta dió los valores más altos encontrados en estas observaciones. Son los que aparecen marcados con una cruz en el gráfico $N .^{\circ} 2$. 
Mauriquand y Gillet, 1935 (21), sometiendo a dieta de carencia a hembras de cuy preñadas, observan que se produce siempre el aborto cuando el embarazo está en la primera mitad:" el organismo de la madre no presenta trastornos y el plazo de aparición del escorbuto experimental se cumple como en los animales testigos. En cambio, si la carencia afecta a las bembras en la segunda mitad de la preñez, el embarazo continúa y la. carencia no se manifiesta, una vez producido el parto, las hembras caen rápidamente en caquexia escorbútica y mueren. Tanto los órganos maternos como los fetales presentan una depleción completa de factor $\mathrm{C}$. Los autores dicen haber encontrado también valores bajos en la placenta, pero no se te fieren a ninguna cifra y aplazan la interpretación de esta experiencia; hasta completarla con nuevos trabajos.

Con lo anteriormente expuesto: relaciones sangre materna, sangre fetal, o del cordón, placenta, podría creerse que la placenta es el órgano "protector" de la experiencia de Mauriquand, puesto que, producido el parto, tanto la madre como el feto, quedan desligados de él y en ambos aparecen los signos de carencia. En apoyo de esta idea está el hecho de que muchas hembras ingieren la masa placentaria, demostrando con esto que se trata de un tejido útil a su organismo.

4) Situación del feto y recién nacido. Defźnsa o resistencia al esçorbuto.

Para analizar las condiciones en que queda el niño después de nacer, se buscaton los valores de ácido cevitamínico en la leche de las madres entre el tercero y noveno día del puerperio, tomando, además, sangre de la vena cada vez que se hacía la titulación en la leche.

La concentración sanguínea se encontró como antes del parto o fuera del embarazo, alrededor de $1 \mathrm{mgr}$. \% en cc. La leche, en cambio, dió valores considerablemente más altos. 


\section{GRAFICO N.甲 3.}

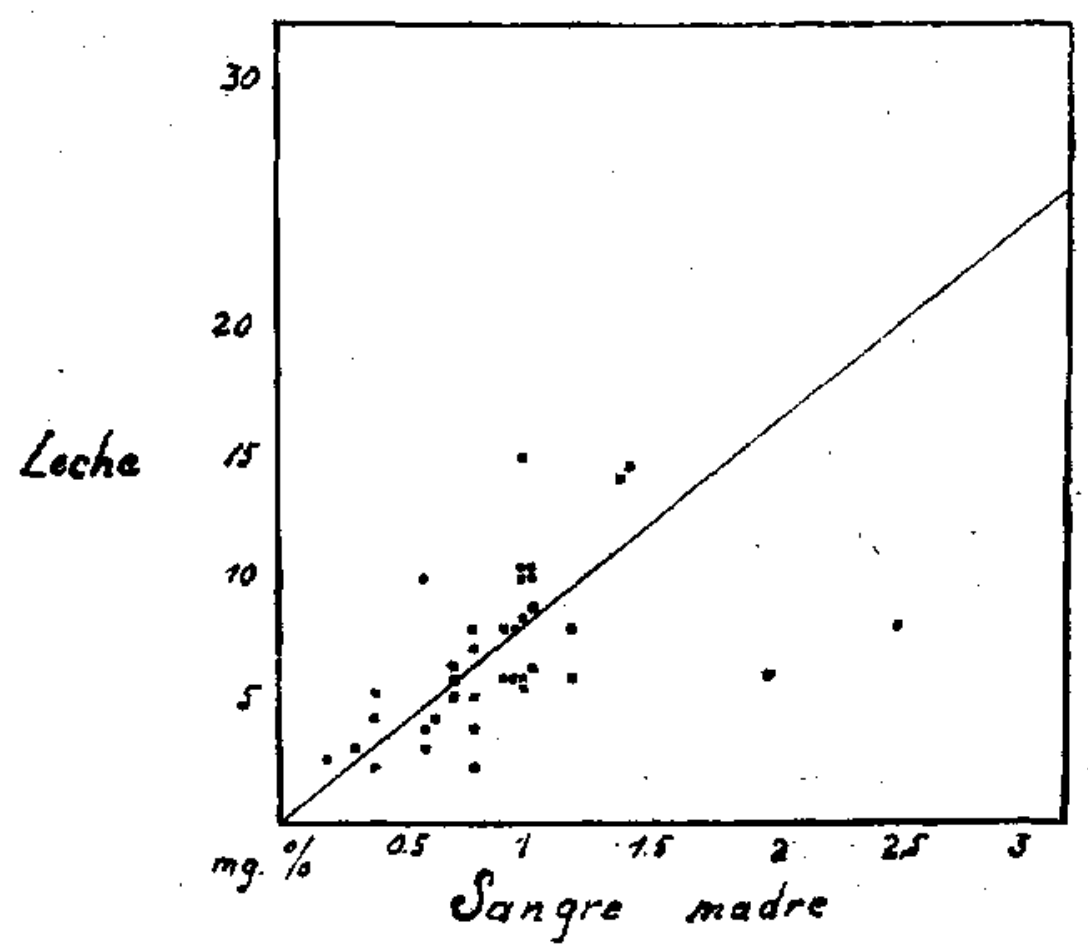
ir leche.

Comparación del contenido de ácido ascórbico en sangre

Término medio: $8,7 \%$ para la leche y $1 \mathrm{mgr}$. \% para la sangre (28 casos).

Pudo establecerse en la mayoría de los casos una evidente relación entre ambos valores que se mantiene alrededor de $1: 8$. En las mujeres cuyos valores sean subnormales en la sangre. el contenido de la leche era también muy bajo. 
CUADRO N. 2.

Contenido de ácido ascórbico en la leche, cuando tos valores en la sangre son sub-normales.

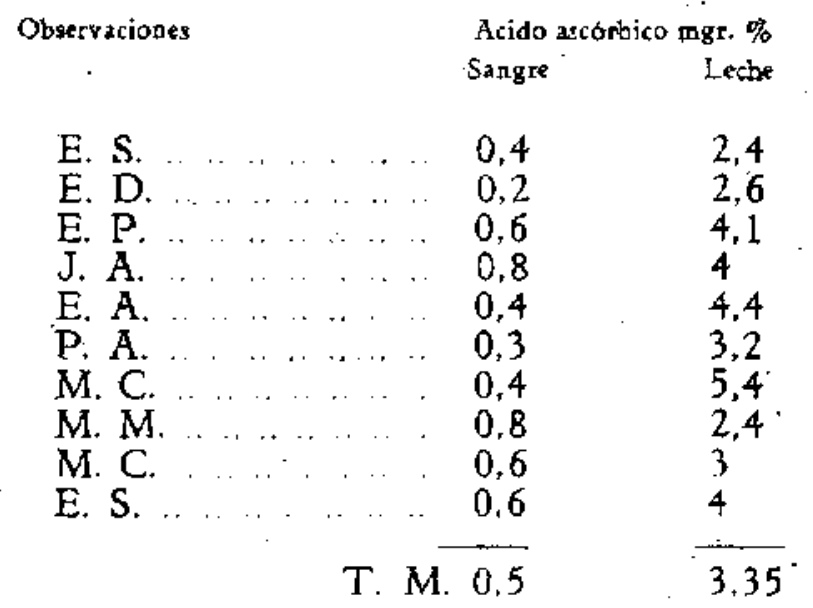

mgr. $\%$ cc.

Tomando en cuenta la cantidad de leche que consume digriamente un lactante sano, 600-700 grs., puede avaluarse ér 48.56 mgrs. la cantidad de ácido cevitamínico que recibe el niño en la leche de su madre al día.

La concèntración alta en la leche respecto al contenido de la sangre supone un proceso análogo al observado en la placenta. El papel del tejido específico secretor consiste en concentrar las sustancias útiles de la sangre y transformarlas hasta hacerlas diferentes de la sustancias original, como sucede, por ejemplo, con la lactosa y la lactalbúmina: para el factor ' $\mathrm{C}$ existiria sólo el proceso de concentración, puesto que es 'una sustancia muy difusible y no sufre transformaciones en los te jidos.

De los datos anotados podría deducirse que el feto y el recién nacido se encuentran naturalmente defendidos de fos estados carenciales a expensas de los importantes aportes que hace el organismo de la madre: durante el embarazo, concentrando factor $C$ en la placenta: durante la lactancia. manteniendo una concentración en la leche. Sin embargo, como lo bace notar Gaethgens, las condiciones del niño yarian respecto de las del feto. Mientras dura el embarazo, la madre entrega sin restricciones sus reservas, asegurando de ese modo el 
aporte al feto; en la lactancia, en cambio. lo hace sólo a medida de su concentración sanguínea: queda el niño a merced del estado de saturación del organismo de su madre. En otros términos: en regimenes pobres, durante el embarazo, la madre está amenazada de sufrir la carencia: durante la lastancia. lo están. la madre y el niño.

La situación de los nifrios que desde el comienzo de su vida quedan privados de alimentación natural, es manifiestamente inferior en este sentido. Baste recordar al respecto que la leche de vaca fresca, contiene sólo $2,5 \mathrm{mgrs}$. $\% \mathrm{cc}$, que después de hervida, desciende a $0,6-1$ : si se agrega que durante los primeros meses, la leche se diluye al $1 ! 2$ o al $1 / 3$ para homologar el contenido en albúmina al de la leche de mujer, resulta que el aporte diario de factor $C$ no pasa de 3-5 mgrs.

Se buscaron también los valores en los alimentos que se usan en la Incubadora de la Maternidad del Salvador, Babeurre y el Lacto al $7 \%$. En la mezcla lista para ser ingerida por los niños se encontró 1,2 mgrs. $\%$. Comparando esta concentración con el término medio de la leche humana. resulta 6.9. veces superior la última; y el aporte total diario es:

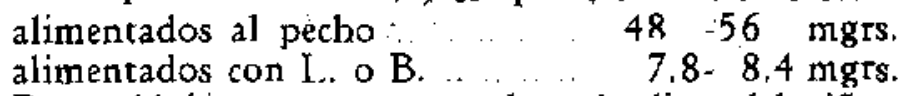

Parece lógico entonces, completar la dieta del niño recién nacido con el aporte de vitamina que falta, o sea altededor de 40 mgrs. diarios, haciendo en esta forma más semejante a la alimentación materna la mezcla que se emplee.

En vista del interés que presentaba poder calcular el contenido sanguíneo de los niños en los primeros días de la vida. se ensayó un micro método basado en el đe Abt y Farmer. 1936 (2), pero con algunas modificaciones. Se procedió en la sigưiente forma: ."

1) Se toma sangte del talón del niño por medio de una lanceta de Francke.

2) 1!10 cc. de sangre total se mezcla con 1,10 ce: de ácido tricloracético al $20 \%$ y con $3 \mid 10 \mathrm{ce}$. de agu destilada.

3) Se centrifuga.

4) Se titula con la solución de diclorofenol indofenol al $2 \%$ por medio de una pipeta de $1 / 10$ de cc. graduada en 100. La titulación se hace sobre todo el centrifugado y se refiere al $1 \mid 10$ de cc.

Se hicieron varios controles frente al método corriente. utilizando sangre de adulto, y en vista de que los resultados. eran perfectamente comparables, se tomaron algunos casos. 
GRAFICO N. 9

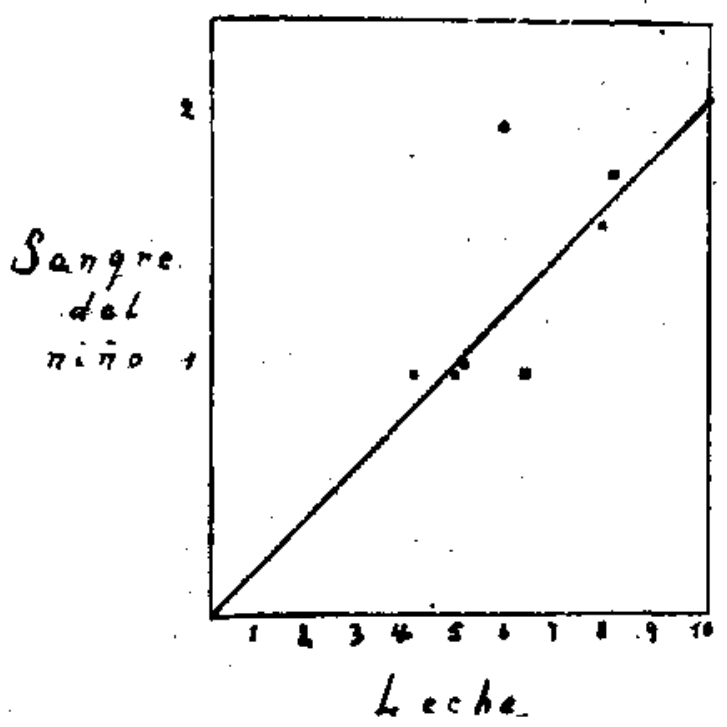

Comparación del contenido de ácido ascórbico en sangre materno, leche y sangre de niños alimentados a pecho exclusivo.

T. M.

$$
\begin{array}{cc}
\quad 0,96 & 1,32 \\
\text { Sangre tnaterna } & \text { Sangr: tel. ziño }
\end{array}
$$

$6,12 \underset{\text { Lethi }}{\operatorname{mgr}} \%$.c.

Como puede observarse, los valores son más alros en la criatura que en su madre y el término medio de estos niños resuita superior también al que se encontró en 1a totalidad de los adultos.

Puede compararse también el contenido total de la sangre circulante en el recién nacido y en el adulto:

Adulto de $65 \mathrm{kgrs}$. peso $5 \mathrm{lts}$. de sangre contienen 51 ingrs. de vitamina $C$.

Niño de 3.300 peso $333 \mathrm{cc}$ de sangre contienen 4.995 mgts. de vitamina $C$.

O sea, la relación de peso del adulto al niño, es de $1: 20$ y la relación del contenido sanguíneo es de $1: 10$. El niño es entonces comparativamente más rico en factor $C$ que el adulto. 
Con el fin de establecer una comparación entte los tecién nacioos y los niños alimentados durante algún tiempo artificialmente, se hizo también la titulación en un grupo de éstos

Se encontraron los siguientes valores:

\section{CUADRO N.93.}

Contenido de ácido ascórbico en sangre de niños alimentados artificialmente.

Nombre Alimentación Pesonx. Pesoac1. Edad Vit. C.

\begin{tabular}{|c|c|c|c|c|}
\hline Alicia & art. & 2.500 & 5.160 & $3 \mathrm{~m} \cdot 13 \mathrm{~d}$. \\
\hline Elsa & & 3,900 & 3.800 & $2 \mathrm{~m}$. \\
\hline Rosa & $"$ & 3.080 & 4.000 & $2 \mathrm{~m} .5 \mathrm{~d}$. \\
\hline Isabel & mixta & 3,500 & 3.470 & $19 \mathrm{~d}$. \\
\hline Pérez & & 2,900 & 2,880 & $2 \mathrm{~m}$ \\
\hline Auristela & " & 3,330 & 4.000 & $11 / 2 \mathrm{~m}$. \\
\hline Herminia & art. & 3,350 & 3.340 & $1 \mathrm{~m}$. \\
\hline
\end{tabular}

Término medio 1.22

El término medio resulta inferior al de los recién nacidos, pero estas cifras no son perfectamente comparable, pues se trata de niños de distintas edades y pesos, lo que s?. guramente influye en el gasto de ácido ascórbico.

Son muy interesantes a este respecto los trabajos de Rowland y Midlin (33), quienes han hecho dosificaciones seriadas inmediatamente después del nacimiento y varios días después. Ellos agrupan a los niños según la alimentación que reciben en tres categorías:

a) Alimentados por sus madres;

b) alimentados artificialmente:

c) alimentados artificialmente, pero con aportes de 30-70 mgrs. de ácido ascórbico diatios.

Encuentran para el primer grupo $1 \mathrm{mgr}$.

$$
\begin{aligned}
& 0,3 \\
& 0,4
\end{aligned}-0.8 \text { respectivamente. }
$$

Concluyen atribuyendo a la leche materna la concen. tracción que podría llamarse "óptima" de los primeros, y re- 
comendando para los alimentados artificialmente el uso de suplementos vitamínicos.

Sybill L. Smith (37), tomando en cuenta la cifra de 5. - 15. mgrs, acordada para el aporte diario al niño en el comité técnico de nutrición de la Liga de las Naciones. la considera demasiado exígua y recomienda el uso de cantida. des bastante mayores. Llega a esta conclusión después de revisar una serie de trabajos que demuestran el rápido descenso del ácido ascórbico en los primeros días de la vida, descenso que no se recupera, si la alimentación sigue siendo pobre en factor C. En este mismo trabajo se cita la observación de Ingalls (37) que comprueba, además del descenso en la sangre, signos claros de carencia en la autopsia de niños criados artificialmente, que fallecieron a los 4 meses de edad. Para este autor tiene gran, importancia la administración de factor $C$ en todos los niños privados de alimentación natural. pero especialmente en los que suften de cualquier cuadro infeccioso o digestivo y en los prematuros.

Así como al estudiar los órganos endocrinos se observa que algunos aumentan o disminuyen su importancia según la edad del individuo. podría decirse que el ácido ascórbico tiene especial importancia durante la niñez y juventud, esto es durante el período del desarrollo. Su presencia es necesaria para la correcta formación de sustancia intercelular en todos los tejidos mesenquímáticos. La deficiencia se manifiesta por fluidez anormal o falta de gelificación de la materia intercelular que es lo que confiere precisamente sus caracteres a las lesiones escorbúticas en los huesos, dientes, cartílagos; no se ha podido establecer histológicamente la lesión vascular, pero se la supone situada en el cemento o bien en la vaina conjuntiva.

Aschoff y Koch, Westin, Fish y Harris (5) coinciden en la observación de la similitud que presentan las lesiones es. corbúticas de los diversos tejidos con los fenómenos que ocurren en la edad senil. Concluyen que estas lesiones pueden interpretarse como involución o vejez prematura $d_{\mathfrak{e}}$ los tejidos.

Las lesiones dentarias son las primeras en aparecer. y preceden incluso a las de los buesos. Walkoff (5), ha encontrado estas lesiones en el escorbuto experimental congénito e induce que deben existir también en el escorbuto infantil. Además, todos los autores insisten en la mayor intensidad de las lesiones en los organismos en crecimiento que tienen más actividad. 
De estas consideraciones thuye la importancia que tiene e1 factor $\mathrm{C}$. durante la formación y crecimiento del nuevo ser: tanto en el organismo joven como en el de su madre, ya que ambos se encuentran en una fase de gran actividad, aportando el uno todo el material que el otro requiere para su perfecto desarrollo. Es precisamente cuando debe datse la atención necesaria a la nutrición de la madre, poniéndola en condiciones de subvenir a todos los requerimientos del niño sin que sufra menoscabo su propio organismo.

Semejante a esta conducta es la indicada para el lactante: no esperar que aparezcan signos anatómicos. que indiquen fallas graves y prolongadas del régimen, sino tratar de preveer todas las necesidades del niño en desartollo, perfeccionando al máximum los aportes alimenticios.

\section{Resumen y conclusiones}

Con el objeto de conocer el contenido sanguineo y los aportes de ácido ascórbico durante el embarazo y la lactancia, se estudió:

1) El contenido en sangre de embarazadas de término, encontrándose una cifra media de $1 \mathrm{mgr}$. $\%$, o sea, semejante a la que se obtiene en adultos normales.

2) Se comparó el contenido en la sangte del cordón con el de la sangre materna, comprobándose que, siendo aqual más elevado, crece paralelamente al contenido de ésta, siondo su promedio 1,2 mgr. \% en el cordón para $1 \mathrm{mgt}$. en la sangre de la madre.

3) El contenido en la placenta se encontró del orden de 10 veces superior al de la sangre, valor que es relativamente constante y no aparece influenciado por el contenido sanguíneo. Este órgano parece fijar electivàmente el ácido ascórbico, aun cuando la tasa sanguínea se mantenga baja (pruebas de sobrecargo). De esto podria deducirse que durante el embarazo, la madre suministra al niño las cantida. des necesarias de factor $C$, actuando la placenta como un reservario de esta sustancia.

4) El contenido de la leche dió cifras variables entre 6 y 10 mgrs. \% cc. (T. M. 8,7) y una relación más o menos constante del orden $8: 1$ respecto a la sangre materna: el aporte diario para el lactante es, por consiguiente, de $40-$ $50 \mathrm{mgrs}$. 
5) En los recién racidos con alimentación natural, se encontraron cifras entre 1 y 2 mgrs. $\%$ cc., siendo el promedio 1,3 ; la sangre de sus madres contenía un promedio de 0,9 mgr. \% cc.

Estos resultados inducen a pensar que el recièn nacido es un organismo sáturado, aun cuando no lo sea su madte, $y$ que esta saturación se mantiene en el niño criado al pecho por los aportes de ácido ascórbico en la leche materna 40 -. 50 mges. diarios, lo que explica la rareza del cuadro carencial en el lactante.

6.) En un escaso número de niños con alimentación attificial, no se encontró un descenso importante (T. M. 1.2) del ácido ascótbico en la sangre, a pesar de que los aportes son muy bajos: $8-10 \mathrm{mgrs}$. diarios. Puede que en ellos exista una carencia latente como se observa durante algún tiémpo en el escorbuto experimental.

7) Debe suministrarse a las madres durante la lactancia y a los niños alimentados artificialmente, los aportes necesarios de factor $C$.

De estos hechos se deduce que para obtener un aporte óptimo de ácido ascórbico en el niño, es necesario que lo sed el de la madre durante el embarazo y lactancia natural, y que en la lactancia artificial es conveniente proporcionar desde un principio una fuente de Vitamina $C$, diversa del régimen utilizado habitualmente.

\section{BIBLIOGRAFIA}

1.-ABASSY, HARRIS L. y otros (1935). -- Lancet. T. 2, pág. 1939.

2.- ABT, FARMER. CHESTER (1936), -- Proc. Soc. Exp. Biol. Me. ., T 34, p. 146 .

3-AGLAR JORDAN (1926) - Fisiología Infantil.

4.-BAUMANN T. RAPOLT L. (1937). - Ztehr. F. Vitamiaforsin, T. 6. pàg. 1 .

5. -EDDY Y DALLLDORF G. (1938). - The avitaminosis.

6.-FELLERS (1935). - A. J. of P. Haelnh N. Y.. T. 25, p. 1340.

7.-GAEHTGENS (1927). — J. A. M. A. T. 108, pág. 1927.

8.-GIROUD A. (1934), - C. R. Soc. Biol., T. 117, pág. 612.

9.-GIROUD A. (1936). - C. R. Soc. Biol. T. 121. pág. 1062.

10.-GIROUD A. (1936). - C. R. Soc. Biol. T. 121. pág. 1580

1 I.-GAGYI (1933). 一 Klin. Wochen. T. 15. pág. 190.

12.-HARRIS L. (1938). - Perspectives in Biochemestry, pág. 329. 
13.-HARRIS L. y RAY (1935). 一 Lancet. T, 1, pág. 7].

14.-HIARRIS L. 7 RAY (1933). - Biochem. J., T. 27. pag. 2011.

15.-INGALlS T. H. (1937). - J. of Péd. T. 10, pág: 577.

16.--INGALLS T. H. (1938), - J. of Ped., T. 56. pág. 1011.

17.-KING C. G. (1938). - J. A. M. A., T. 111 , pág. 1462.

18.-KING C. G. (1938). - J. A. M. A., T. 111. p. 1098.

19.-MAURIQUAND G. GiLLET R. y COEUR A. (1936). - C. R. So." Bioll. T. 117. pág 1230.

20.- MAURIQUAND G. GLLUET R. y COEUR A. (1936), - C. R. SEc Biol.. T. 121, pág. 1064.

21. -MAURIQUAND G. GLLLET R. y'COELR A, (1935) - C. R. So: Biol., T. 120, pág. 214.

22,- MAUIRIQUAND G. GILLET R. y COEUR A. $\{1936\rangle,-$ C. R. $5 r i$ Biol. T. 121. pág. 216.

23.-MAURIQUAND G. GILLET R. y COEUR A. (1936). - C. R. 5n. Bíol., T. 123, pág. 1038.

24.-MAURIQUAND G. y TETE H. y otros $(1937)$ - Prse Med. T. 35. par. 657.

25.-WXEYER F. y NASSAU E. (1935). - Alim:atación del niño de peço.

26.-NEUWEILER W. (1935). - Klin, Wochen. T. 14, pág. 1040.

77. -NEUWEILER W. (1935). - Klín Wochen., T. 14, pág. 1041

28. -NEUWEILER W. (1935). - Klin Wodken., T. 14, pajg. 1793

29.- NEUWEILIER W. (1935), - Zeit:chr. F. Vitaminforsch. T. 4. pág. 39.

30.-NEUWEILER W. (193\%), - Zeitgchr. F, Vitaminforsch. T. 6. pág. 75.

31.-NEUWEILER W. (1938). -. Presse Med., T. 37. pág. 734.

32-ROHMMER P. y BESZONIFF N. (1936). - C. R. Sor. Biol. T. 121. pág. 988.

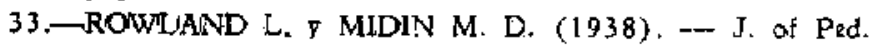

34.-SCHOEDER. H. (1935). - Klin. Wochen., T. 14, paig. 484.

25.-SZENT GYORGYI A. (1938). - Presse Meć., T. 995, pás 31.

36. -STEPP W. y SCHOEDER H. (1935). -- Klin, Woh:n, T. I4, pág. 147.

3.7.-SIMITH S. L. (1938). - J. A. M. A., T. 111 , pág. 1753

38. - TAUBER H. (1937). - Enzime Oheme:try. pág. 188.

39.-TEEE H. M. y otros (1938). - A. J. of D. af Child. T. 56. pág. 104.

40.-TIOROK G. y NEUFELD L. (1936). - Klín. Woch:n.. T. 15, pág. 477 . 